\title{
PROGNOSTIC PERFORMANCE OF WORLD SOCIETY OF EMERGENCY SURGERY SEPSIS SEVERITY SCORE IN BULGARIAN PATIENTS WITH COMPLICATED INTRA-ABDOMINAL INFECTIONS
}

\author{
E. Dimitrov*, G. Minkov, E. Enchev, Y. Yovtchev \\ Department of Surgical Diseases, University Hospital "Prof. Dr St. Kirkovich”, Stara Zagora, \\ Bulgaria
}

\begin{abstract}
PURPOSE: To evaluate the ability of World Society of Emergency Surgery Sepsis Severity Score (WSES SSS) to prognosticate the fatal outcome in our local setting and compare the findings with global data.

METHODS: The retrospective study involved 110 patients with complicated intra-abdominal infections (cIAIs) admitted to Department of Surgical Diseases at a University hospital. All adult patients who required emergency surgery due to cIAIs from January 2017 to July 2019 were included. We assessed the prognostic performance of WSES SSS using area under receiver operating characteristics (AUROC) curves and analyzed the coordinates of the curves.

RESULTS: The observed in-hospital mortality was $22.7 \%$. WSES SSS was significantly higher in nonsurvivors compared to survivors - 7(5-8) points vs. $3(0-5)$ points, $p<0.0001$. The established AUROC value of WSES SSS for outcome prediction was $0.825(0.749-0.902)$. We have identified sensitivity of $92 \%$ and specificity of $68.2 \%$ for cut-off value $>4$ points.

CONCLUSION: The WSES SSS was shown as a practical and reliable mortality predictor in Bulgarian patients with cIAIs.
\end{abstract}

Key words: WSES SSS, intra-abdominal infections, outcome, mortality

\section{INTRODUCTION}

In the third decade of the twenty-first century, complicated intra-abdominal infections (cIAIs) still represent a challenge for surgeons and intensive care physicians. The cIAIs are infections that spread beyond the affected intraabdominal organ, and result either in local or diffuse peritonitis [1]. They are responsible for about one fifth of sepsis in intensive care units (ICUs), and are associated with high morbidity and mortality [2].

Prognostic assessment at early stage of patients with cIAIs could help for the differentiation of those at a higher risk of death and provides an opportunity to change the management strategy, which might affect the adverse

Correspondence to: Evgeni N. Dimitrov, M.D. Department of Surgical Diseases, University Hospital "Prof. Dr. Stoyan Kirkovich" Stara Zagora, 2 Gen. Stoletov Str. Bulgaria; e-mail: evgeni_d1984@yahoo.com tel: +359887609943 outcome. Over the years, various prognostic scores have been developed, however none of them is widely accepted in everyday practice. Many of these scores have proven to be reliable, but time consuming, difficult to calculate and complex; they require many laboratory and clinical data, and outside ICUs are rarely used.

The most recent and least investigated surgical score is the WSES Sepsis Severity Score (WSES SSS). It was designed by the World Society of Emergency Surgery (WSES) in 2014 as a prognostic scoring system specific for cIAIs [3]. Several studies validated this score globally [4-6] and found that it can be precise and practical for patients with cIAIs. A major advantage of WSES SSS is its simplicity and easy calculation. No study (to the best of our knowledge) has evaluated this score in Bulgarian patients yet. 
Therefore, the aim of our study was to analyze the ability of WSES SSS to predict mortality in patients with cIAIs in our local setting.

\section{MATERIAL AND METHODS}

We conducted a retrospective study at a University Hospital "Prof. Dr. Stoyan Kirkovich" Stara Zagora. The medical records of 110 adult patients admitted to the Department of Surgical Diseases (DSD) from the Emergency Department and operated on for
cIAIs between January 2017 and July 2019 were reviewed. For the time period, 131 patients with a diagnosis cIAI were admitted to DSD. In 18 patients we found missing data on some clinical parameters, 2 patients died before surgery, and one was $<18$ years old. Finally, demographic data, clinical information, and clinical outcomes were determined from 110 patients' medical records. The WSES SSS was calculated based on 6 risk factors postoperatively [4] (Table 1).

Table 1. WSES Sepsis Severity Score (0-18 score)

\begin{tabular}{|l|l|}
\hline Risk factor & Points \\
\hline Age $>$ 70 years & 2 \\
\hline Immunosuppression & 3 \\
\hline Setting of acquisition & 2 \\
\hline Healthcare-associated infection & \multicolumn{2}{|l|}{} \\
\hline Clinical condition at admission & 3 \\
\hline Severe sepsis & 5 \\
\hline Septic shock & 2 \\
\hline Origin of cIAIs & 2 \\
\hline Colonic non-diverticular perforation peritonitis & 2 \\
\hline Diverticular diffuse peritonitis & 3 \\
\hline Postoperative diffuse peritonitis & \\
\hline Small bowel perforation peritonitis & 3 \\
\hline Delay in source control & \\
\hline Delayed initial intervention > 24 hours &
\end{tabular}

The obtained data were analyzed using SPSS Statistics 19.0 (IBM, Chicago, Illinois, USA). The ability of WSES SSS to prognosticate the fatal outcome was evaluated using Receiver Operating Characteristic (ROC) Curve Analysis and direct logistic regression model. Continuous variables were presented as mean $( \pm \mathrm{SD})$ for normally distributed data or median (IQR) for non-normally distributed data. Comparisons of group differences for continuous variables were performed by Student $t$ Test or Mann-Whitney $U$ test. Categorical variables were expressed as frequency (\%) and compared by Chi-square test or Fisher exact test. P-value was considered significant at $<0.05$.

\section{RESULTS}

\section{Patients' characteristics}

Of the 110 patients, 25 (22.7\%) had an adverse outcome. None of the patients was immunosuppressed, had septic shock or healthcare-associated cIAIs. Survivors were significantly younger than non-survivors $(56.84 \pm 18.89$ vs. $74.80 \pm 12.64, p<0.0001)$. The most common cause of cIAIs was acute appendicitis (27\%), followed by acute cholecystitis and peptic ulcer perforation. In patients with chronic renal failure $(\mathrm{p}=0.004)$ and malignancy $(\mathrm{p}=0.002)$ mortality rate was significantly higher. We found significant differences between survivors and nonsurvivors according to exudate $(p=0.007)$, spread $(\mathrm{p}=0.016)$ and source of peritonitis $(\mathrm{p}=$ 0.041 ). Patients who had severe sepsis at admission ( $p<0.0001)$ or underwent delayed initial intervention $>24$ hours had higher risk of death $(\mathrm{p}=0.004)$ (Table 2).

\section{WSES SSS}

Median WSES SSS of the sample was 3 (0-7) points. Non-survivors had a significantly higher median score than survivors -7 (5-8) vs. 3 (05), $\mathrm{p}<0.0001$ (Figure 1). The highest calculated score was 10 points, and thirty-four patients had WSES SSS $=0$ points. WSES SSS $>4$ points was observed in 31.8 percent of survivors and 23 out of 25 of non-survivors (Table 2). 


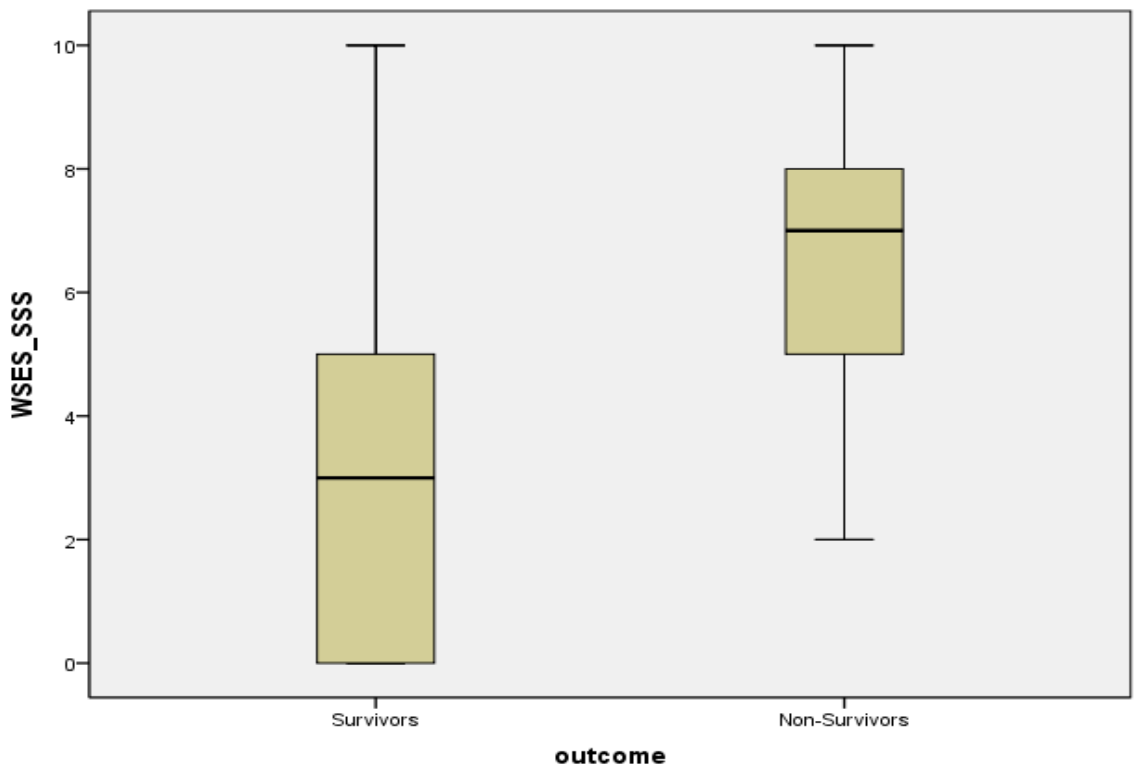

Figure 1. Boxplot of WSES SSS in survivors and non-survivors

Table 2. Patients' characteristics

\begin{tabular}{|l|l|l|l|l|}
\hline Variable & Total population & Survivors(n=85) & $\begin{array}{l}\text { Non- } \\
\text { Survivors(n=25) }\end{array}$ & p value \\
\hline Sex, n(\%) male/female & $61(55.5) / 49(45.5)$ & $48(78.7) / 37(75.5)$ & $13(21.3) / 12(24.5)$ & 0.693 \\
\hline Age, years \pm SD & $60.92 \pm 19.17$ & $56.84 \pm 18.89$ & $74.80 \pm 12.64$ & $<0.0001$ \\
\hline Age $>$ 70 years, $\mathbf{n}(\%)$ & $46(41.8)$ & $27(31.8)$ & $19(76.0)$ & $<0.0001$ \\
\hline $\begin{array}{l}\text { Source, } \mathbf{n}(\%) \\
\text { Appendix }\end{array}$ & $27(24.5)$ & $25(29.4)$ & $2(8.0)$ & 0.041 \\
Hepatobiliary system & $26(23.6)$ & $20(23.5)$ & $6(24.0)$ & \\
Stomach/duodenum & $24(21.8)$ & $18(21.2)$ & $6(24.0)$ & \\
Colon/Rectum & $18(16.4)$ & $10(11.8)$ & $8(32.0)$ & \\
Small intestine & $2(18)$. & $1(1.2)$ & $1(4.0)$ & \\
Gynecological & $7(6.4)$ & $7(8.2)$ & $0(0)$ & \\
Other & $6(5.5)$ & $4(4.7)$ & $2(8.0)$ & \\
\hline Peritonitis, n(\%) & & & & \\
Local & $40(36.4)$ & $36(42.4)$ & $4(16.0)$ & \\
Diffuse & $70(63.6)$ & $49(57.6)$ & $21(84.0)$ & \\
\hline Exudate, $\mathbf{n}(\%)$ & & & & \\
Serous & $21(19.1)$ & $19(22.4)$ & $2(8.0)$ & \\
Purulent & $84(76.4)$ & $65(76.5)$ & $19(76.0)$ & \\
Feculent & $5(4.5)$ & $1(1.2)$ & $4(16.0)$ & \\
\hline Comorbidity,n(\%) & & $32(37.6)$ & $12(48.0)$ & 0.007 \\
High Blood Pressure & $44(40.0)$ & $7(8.2)$ & $9(36.0)$ & 0.002 \\
Malignancy & $16(14.5)$ & $10(11.8)$ & $3(12.0)$ & 1.000 \\
Diabetes & $13(11.8)$ & $3(3.5)$ & $6(24.0)$ & 0.004 \\
\hline Chronic Renal Failure & $9(8.2)$ & $37(43.5)$ & $19(76.0)$ & 0.004 \\
\hline Delayed intervention $>\mathbf{2 4 h}$ & $56(50.9)$ & $21(24.7)$ & $16(64.0)$ & $<0.0001$ \\
\hline Severe sepsis & $37(33.6)$ & $3(0-5)$ & $7(5-8)$ & $<0.0001$ \\
\hline WSES SSS, points(IQR) & $3(0-7)$ & $27(31.8)$ & $23(92.0)$ & 0.0001 \\
\hline WSES SSS >4, n(\%) & $50(45.5)$ & & \\
\hline
\end{tabular}

\section{Sensitivity, Specificity and AUROC}

WSES SSS showed a good ability to prognosticate the fatal outcome (AUROC = $0.825,95 \% \mathrm{CI}=0.749-0.902, \mathrm{p}<0.0001)$. The optimal cut-off value that we determined was WSES SSS > 4 points and it permitted prediction of mortality with a sensitivity of $92 \%$ and a specificity of $68.2 \%$ (Table 3) (Figure 2.) 
Table 3. Sensitivity, Specificity and AUROCs

\begin{tabular}{|l|l|l|l|l|}
\hline Variable & Sensitivity,\% & Specificity,\% & $\begin{array}{l}\text { AUROC (95\% } \\
\text { CI) }\end{array}$ & Significance \\
\hline WSES SSS $>$ 4 & 92.0 & 68.2 & $0.825(0.749-$ & $\mathrm{p}<0.0001$ \\
\hline WSES SSS $>$ 5 & 68.0 & 80.0 & $0.902)$ & \\
\hline WSES SSS $>$ 6 & 56.0 & 82.4 & & \\
\hline WSES SSS $>$ 7 & 44.0 & 84.7 & \\
\hline
\end{tabular}

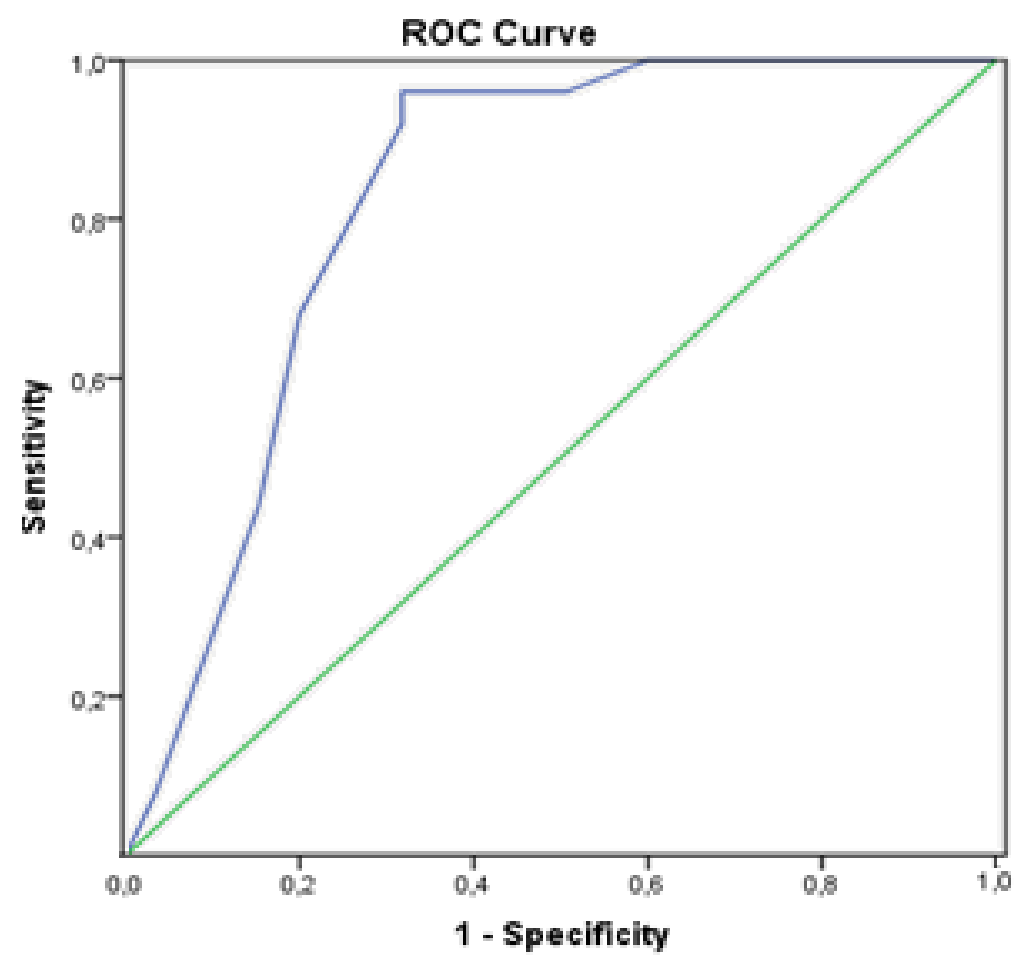

Figure 2. ROC Curve

\section{Logistic regression}

The ability of WSES SSS to prognosticate death was tested by direct logistic regression (Table 4). This model using only the WSES SSS was highly significant $(\mathrm{p}<0.0001)$. The odds of unfavorable outcome increased by 0.535 by an increase on 1 point of the score, which is notable.

Table 4. Direct logistic regression model for mortality prediction

\begin{tabular}{|l|l|l|l|l|l|}
\hline Variable & B & S.E. & Wald test & p-value & Odd Ratio (95\% CI) \\
\hline WSES SSS & 0.428 & 0.097 & 19.332 & $<0.0001$ & $1.535(1.268-1.858)$ \\
\hline
\end{tabular}

\section{DISCUSSION}

Complicated intra-abdominal infections are still associated with unacceptably high morbidity, mortality, and healthcare costs globally [3, 4, 9]. Prognostic evaluation of cIAIs at early stage of the disease and timely treatment can improve the poor outcome, which indicates the necessity of reliable tools for easier identification of patients at a higher risk of death. A number of researchers are trying to deal with these problems by developing novel prognostic scoring systems or validating already existing ones [4-10].

The aim of each novel predictive score is to improve the evaluation of a disease and eventually to replace the existing scoring systems. In this regard, WSES developed in 2014 a new clinical scoring system - a WSES Sepsis Severity Score (WSES SSS), which shows a very good ability to predict mortality and can be used worldwide [3]. In 2015 the WISS study [4] confirmed these findings and 
indicated that the best threshold for predicting death was WSES SSS $>5$ with a sensitivity and a specificity of $89.2 \%$ and $83.5 \%$, respectively. Raimondo et al. [7] observed in sixty-five patients with cIAIs an identical cut-off value with sensitivity and a specificity of $85.7 \%$ and $75.9 \%$, respectively. We identified a cut-off value of WSES SSS $>4$ points with a sensitivity of $92 \%$ and a specificity of $68.2 \%$. The same cut-off value was established by Godinez-Vidal et al. [6] in 185 patients with cIAIs and Sazhin et al. [8] in 153 patients with diffuse peritonitis. For a threshold $>4$ points Godinez-Vidal et al. [6] reported a sensitivity and a specificity of $76.47 \%$ and $90.48 \%$, respectively and Sazhin et al. [8] reported a sensitivity and a specificity of $78.6 \%$ and a $84.7 \%$, respectively. Mwenda et al. [5] observed in 173 patients with cIAIs a cut-off value $>6$ with a sensitivity of $80 \%$ and a specificity of $20.9 \%$. The highest threshold WSES SSS $\geq 8$ with a sensitivity of $73 \%$ and a specificity of $76 \%$ was established by Tolonen et al. [9] in 93 patients with severe cIAIs.

In the present study we used ROC curve analysis to determine the threshold values, and the AUROC was tested for significance. The established AUROC was 0.825, which was statistically significant for prediction of unfavorable outcome $(\mathrm{p}<0.0001)$. With the exception of Tolonen et al. [9] (AUROC = 0.809), all other researchers found a higher AUROC of WSES SSS than our study: Godinez-Vidal et al. [6] - AUROC $=0.931$, Raimondo et al. [7] - AUROC $=0.887$, Mwenda et al. [5] - AUROC $=0.874$ and Sazhin et al. [8] - $\mathrm{AUROC}=0.851$.

Non-survivors in our study have significantly higher score than survivors, whereat a threshold $>4$ points is associated with an increased risk of death. We observed that advanced age and delayed intervention are significant prognostic factors $-76 \%$ of patients who died were $>70$ years old and had preoperative duration of peritonitis $>24$ hours. In our study patients with severe sepsis $(\mathrm{p}<0.0001)$, malignancy $(\mathrm{p}=$ $0.002)$ or chronic renal failure $(\mathrm{p}=0.004)$ had a less chance of survival. Using direct logistic regression model we demonstrated that WSES SSS is highly significant prognostic score ( $\mathrm{p}<$ 0.0001 ) with odds ratio of 1.53 .

Sartelli et al. [3] reported a mortality rate of $10.5 \%$ in CIAWO study, and $9.2 \%$ in the WISS study [4]. We observed a much higher mortality rate $-22.7 \%$. This could be due to the fact that in our study most of the patients were presented with diffuse peritonitis $-63.4 \%$ unlike CIAWO $(43.6 \%)$ and WISS (35.9\%) studies. Furthermore, only $24.5 \%$ of the surveyed patients had acute appendicitis as a source of infection, whereby the observed mortality rate was low. In CIAWO and WISS studies patients with acute appendicitis represented $33.3 \%$ and $34.2 \%$ of the population, respectively. In patients with severe cIAIs, Tolonen et al. [9] found a similar mortality rate $-22 \%$. The lower rate was established by Mohan et al. [10] - 16\% in patients with perforation peritonitis, Sazhin et al. [8] - $13.7 \%$ in patients with diffuse peritonitis, Mwenda et al. [5] $-12.8 \%$ and Raimondo et al. [7] $-10.8 \%$ in patients with cIAIs. Godínez-Vidal et al. [6] reported the lowest mortality rate of $9.2 \%$ in patients with cIAIs.

This is the first study, (to the best of our knowledge) that analyzes the prognostic performance of WSES SSS in Bulgarian patients with cIAIs.

As limitations of our study we can highlight the single-center experience, the retrospective design, and the small sample size.

\section{CONCLUSION}

In Bulgarian patients with cIAIs WSES SSS was observed as a reliable and independent predictor of mortality. It allows the surgeon to evaluate the risk of unfavorable outcome even intraoperatively and maybe after more largescale validation studies WSES SSS should be applied routinely in clinical practice.

\section{REFERENCES}

1. Menichetti F, Sganga G. Definition and classification of intra-abdominal infections. J Chemother. 21 Suppl 1:3-4, 2009;

2. Vincent JL, Rello J, Marshall J, et al. International study of the prevalence and outcomes of infection in intensive care units. JAMA, 302(21):2323-2329, 2009;

3. Sartelli M, Catena F, Ansaloni L, et al. Complicated intra-abdominal infections worldwide: the definitive data of the CIAOW Study. World J Emerg Surg. 9:37, 2014;

4. Sartelli M, Abu-Zidan FM, Catena F, et al. Global validation of the WSES Sepsis Severity Score for patients with complicated intra-abdominal infections: a 
prospective multicentre study (WISS Study). World J Emerg Surg. 10:61, 2015;

5. Mwenda KI, Daniel O, Mark A. Utility of World Society of Emergency Surgery Sepsis Severity Score in Predicting Outcomes of Intra-abdominal Infections. The ANNALS of AFRICAN SURGERY, 17(2) 65-68, 2020;

6. Godínez-Vidal AR, Vázquez-Rentería R, Guerrero-Ponce AE, et al. Use of the WSES scale to predict mortality in patients with intra-abdominal infection. Rev Mex de Cirugía del Aparato Digestivo. 9(2): 6570, 2020;

7. Raimondo S., Sartelli M., Coccolini F., et al. Which prognostic score for abdominal sepsis? Analysis of final results of PIPAS (Physiological Indicators for Prognosis in Abdominal Sepsis) study in a single center. Journal of Peritoneum (and other serosal surfaces). 3:106, 2018;
DIMITROV E., et al.

8. Sazhin A, Ivakhov G, Stradymov E,et al. The modified Boey score (mBoey) for outcome prediction in patients with perforated peptic ulcer complicated by diffuse peritonitis: a retrospective study. Research Square; preprint 2020;

9. Tolonen M, Coccolini F, Ansaloni L, et al. Getting the invite list right: a discussion of sepsis severity scoring systems in severe complicated intra-abdominal sepsis and randomized trial inclusion criteria. World $J$ Emerg Surg. 13:17, 2018;

10. Mohan R, Sundararajan G. Comparison of WSES Prognostic Score and Mannheim Peritonitis Index in Predicting Outcome of Patients with Peritonitis Secondary to Hollow Viscus Perforation. IOSR Journal of Dental and Medical Sciences (IOSRJDMS). 18(9); 29-31, 2019; 